\title{
LEFT $\ell_{1}$-FACTORABLE POLYNOMIALS
}

\author{
RAFFAELLA CILIA \\ Dipartimento di Matematica, Facoltà di Scienze, \\ Università di Catania, Viale Andrea Doria 6, 95125 Catania, Italy \\ e-mail: cilia@dmi.unict.it \\ and JOAQUÍN M. GUTIÉRREZ \\ Departamento de Matemática Aplicada, ETS de Ingenieros Industriales, \\ Universidad Politécnica de Madrid, C. José Gutiérrez Abascal 2, 28006 Madrid, Spain \\ e-mail:jgutierrez@etsii.upm.es
}

(Received 26 April 2008; revised 30 December 2008; accepted 17 April 2009)

\begin{abstract}
A polynomial $P \in \mathcal{P}\left({ }^{k} E, F\right)$ is left $\ell_{1}$-factorable if there are a polynomial $Q \in \mathcal{P}\left({ }^{k} E, \ell_{1}\right)$ and an operator $L \in \mathcal{L}\left(\ell_{1}, F\right)$ such that $P=L \circ Q$. We characterise the Radon-Nikodým property by the left $\ell_{1}$-factorisation of polynomials on $L_{1}(\mu)$. We study the left $\ell_{1}$-factorisation of nuclear, compact and Pietsch integral polynomials. For Pietsch integral polynomials, we introduce the left integral $\ell_{1}$-factorisation property, obtaining a second polynomial characterisation of the Radon-Nikodým property and showing that it plays a role somehow comparable, in this setting, to nuclearity of operators. A characterisation of $\mathscr{L}_{1}$-spaces is also given in terms of the left compact $\ell_{1}$-factorisation of polynomials.

2000 Mathematics Subject Classification. Primary 46G25; secondary 47H60, 46B20, 46B22.
\end{abstract}

1. Introduction. The following result of Lewis and Stegall relates the RadonNikodým property with the $\ell_{1}$-factorisation of (linear bounded) operators.

THEOREM 1.1. [18, Theorem III.1.8] A Banach space F has the Radon-Nikodým property if and only if for every finite measure space $(\Omega, \Sigma, \mu)$, every operator $T$ : $L_{1}(\mu) \rightarrow F$ admits a factorisation $T=L \circ S$

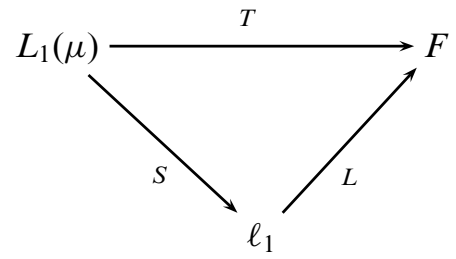

where $S: L_{1}(\mu) \rightarrow \ell_{1}$ and $L: \ell_{1} \rightarrow F$ are operators. Moreover, we have

$$
\|T\|=\inf \|S\|\|L\|,
$$

where the infimum is taken over all such factorisations. 
In particular, let $T: L_{1}(\mu) \rightarrow F$ be a weakly compact operator. Since $T$ factors through a reflexive Banach space [18, Corollary VIII.4.9] and reflexive Banach spaces have the Radon-Nikodým property, $T$ is also $\ell_{1}$-factorable.

We say that a polynomial $P \in \mathcal{P}\left({ }^{k} E, F\right)$ is left $\ell_{1}$-factorable if there are a polynomial $Q \in \mathcal{P}\left({ }^{k} E, \ell_{1}\right)$ and an operator $L: \ell_{1} \rightarrow F$ such that $P=L \circ Q$ :

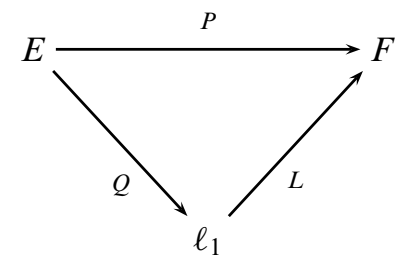

In this case, we denote

$$
\gamma_{\ell_{1}}^{\text {left }}(P):=\inf \|Q\|\|L\|
$$

where the infimum is taken over all such factorisations.

In the present work, we give conditions for a polynomial to be left $\ell_{1}$-factorable. The right $\ell_{1}$-factorisation is defined in a 'symmetric' way and will be considered in a forthcoming paper.

We prove that for every nuclear polynomial $P \in \mathcal{P}_{\mathrm{N}}\left({ }^{k} E, F\right)$, there are a nuclear polynomial $Q \in \mathcal{P}_{\mathrm{N}}\left({ }^{k} E, \ell_{1}\right)$ and an operator $L: \ell_{1} \rightarrow F$ such that $P=L \circ Q$. Moreover, the norms satisfy

$$
\|P\|_{\mathrm{N}}=\inf \|Q\|_{\mathrm{N}}\|L\|,
$$

where the infimum is taken over all such factorisations.

We show that a similar result holds for Pietsch integral polynomials when the range space $F$ has the Radon-Nikodým property. Moreover, this left integral $\ell_{1}$-factorisation property of polynomials characterises the Radon-Nikodým property and replaces somehow in this theory the nuclearity of operators.

A result similar to Theorem 1.1 is obtained for polynomials on $L_{1}(\mu)$, giving another polynomial characterisation of the Radon-Nikodým property.

Finally, we show that a compact polynomial on an $\mathscr{L}_{1}$-space factors compactly through $\ell_{1}$, and this property characterises $\mathscr{L}_{1}$-spaces.

The paper is organised as follows: Section 1 contains introductory material and preparatory results. Section 2 studies the relationship of the left $\ell_{1}$-factorable polynomials with other ideals of polynomials (mainly nuclear and compact polynomials). In Section 3 we introduce and study the left integral $\ell_{1}$-factorisation property. We show that it characterises the Radon-Nikodým property, and we give a result on composition of polynomials and operators, which improves previous work by the authors.

Throughout, $E, F, G, X$ and $Y$ denote Banach spaces, $E^{*}$ is the dual of $E$ and $B_{E}$ stands for its closed unit ball. The closed unit ball $B_{E^{*}}$ will always be endowed with the weak-star topology. By $\mathbb{N}$ we represent the set of all natural numbers and by $\mathbb{K}$ the scalar field (real or complex). By $E \equiv F$, we mean that the Banach spaces $E$ and $F$ are isometrically isomorphic, while $E \simeq F$ means that $E$ and $F$ are isomorphic.

We use the symbol $\mathcal{L}(E, F)$ for the space of all (linear bounded) operators from $E$ into $F$ endowed with the operator norm. Its subspace of compact operators is denoted by $\mathcal{K}(E, F)$. Given a space $F$, we shall denote by $j_{F}$ the natural isometric embedding of 
$F$ into its bidual $F^{* *}$. The sequence $\left(e_{n}\right)_{n=1}^{\infty}$ represents the canonical unit vector basis of $\ell_{1}$.

Given $k \in \mathbb{N}$, we denote by $\mathcal{P}\left({ }^{k} E, F\right)$ the space of all $k$-homogeneous (continuous) polynomials from $E$ into $F$ endowed with the supremum norm. When $F$ is omitted, it is understood to be the scalar field. Recall that with each $P \in \mathcal{P}\left({ }^{k} E, F\right)$ we can associate a unique symmetric $k$-linear mapping $\widehat{P}: E \times \stackrel{(k)}{.} \times E \rightarrow F$ so that

$$
P(x)=\widehat{P}(x, \stackrel{(k)}{.}, x) \quad(x \in E) .
$$

For the general theory of multilinear mappings and polynomials on Banach spaces, we refer the reader to [19] and [29]. The notion of ideal of vector-valued polynomials may be seen, for instance, in [24] and [8].

We use the notation $\otimes^{k} E:=E \otimes \stackrel{(k)}{.} \otimes E$ for the $k$-fold tensor product of $E, E \otimes_{\pi} F$ for the completed projective tensor product of $E$ and $F$ and $\otimes_{\pi}^{k} E$ (respectively $\otimes_{\epsilon}^{k} E$ ) for the space $\otimes^{k} E$ endowed with the completed projective (respectively injective) tensor norm (see [18] for the theory of tensor products). By $\otimes_{s}^{k} E:=E \otimes_{s}{ }_{(k)}{ }^{k} \otimes_{s} E$ we denote the $k$-fold symmetric tensor product of $E$, that is the set of all elements $u \in \otimes^{k} E$ of the form

$$
u=\sum_{j=1}^{n} \lambda_{j} x_{j} \otimes \stackrel{(k)}{.} \otimes x_{j} \quad\left(n \in \mathbb{N}, \lambda_{j} \in \mathbb{K}, x_{j} \in E, 1 \leq j \leq n\right)
$$

By $\otimes_{\pi, s}^{k} E$ (respectively $\otimes_{\epsilon, S}^{k} E$ ) we represent the space $\otimes_{s}^{k} E$ endowed with the topology induced by that of $\otimes_{\pi}^{k} E$ (respectively $\otimes_{\epsilon}^{k} E$ ). For symmetric tensor products, the reader is referred to [21].

By

$$
\delta_{k}: E \longrightarrow \otimes_{\pi, s}^{k} E, \quad \text { or } \quad \delta_{k}: E \longrightarrow \otimes_{\pi}^{k} E, \quad \text { or } \quad \delta_{k}: E \longrightarrow \otimes_{\epsilon, s}^{k} E,
$$

we denote the canonical $k$-homogeneous polynomial given by

$$
\delta_{k}(x):=x \otimes \stackrel{(k)}{.} \otimes x \quad(x \in E) .
$$

If $A: E_{1} \times \cdots \times E_{k} \rightarrow F$ is a $k$-linear (continuous) mapping, the linearisation of $A$ is the operator

$$
\bar{A}: E_{1} \otimes_{\pi} \cdots \otimes_{\pi} E_{k} \longrightarrow F
$$

given by

$$
\bar{A}\left(\sum_{j=1}^{n} x_{1, j} \otimes \cdots \otimes x_{k, j}\right)=\sum_{j=1}^{n} A\left(x_{1, j}, \ldots, x_{k, j}\right)
$$

for all $x_{i, j} \in E_{i}(1 \leq i \leq k, 1 \leq j \leq n)\left[31\right.$, p. 24]. For a polynomial $P \in \mathcal{P}\left({ }^{k} E, F\right)$, its linearisation

$$
\bar{P}: \otimes_{\pi, s}^{k} E \longrightarrow F
$$


is the operator given by

$$
\bar{P}\left(\sum_{j=1}^{n} \lambda_{j} x_{j} \otimes \stackrel{(k)}{.} \otimes x_{j}\right)=\sum_{j=1}^{n} \lambda_{j} P\left(x_{j}\right)
$$

for all $x_{j} \in E$ and $\lambda_{j} \in \mathbb{K}(1 \leq j \leq n)$.

Given a polynomial $P \in \mathcal{P}\left({ }^{k} E, F\right)$, its adjoint is the operator

$$
P^{*}: F^{*} \longrightarrow \mathcal{P}\left({ }^{k} E\right)
$$

defined by $P^{*}(\psi)(x):=\psi \circ P(x)$, for $x \in E$ and $\psi \in F^{*}$. Note that up to the isomorphism $\left(\otimes_{\pi, s}^{k} E\right)^{*} \simeq \mathcal{P}\left({ }^{k} E\right)$, the adjoints $P^{*}$ and $(\bar{P})^{*}$ coincide.

A polynomial $P \in \mathcal{P}\left({ }^{k} E, F\right)$ is (weakly) compact if $P\left(B_{E}\right)$ is a relatively (weakly) compact subset of $F$. The space of compact polynomials from $E$ into $F$ is denoted by $\mathcal{P}_{\mathrm{K}}\left({ }^{k} E, F\right)$. We denote by $\mathcal{P}_{\mathrm{wb}}\left({ }^{k} E, F\right)$ the space of polynomials from $E$ into $F$ whose restrictions to bounded subsets of $E$ are weakly continuous. It is well known that $\mathcal{P}_{\text {wb }}\left({ }^{k} E, F\right) \subseteq \mathcal{P}_{\mathrm{K}}\left({ }^{k} E, F\right)$ (see [6, Lemma 2.2] and [5, Theorem 2.9]).

A polynomial $P \in \mathcal{P}\left({ }^{k} E, F\right)$ is nuclear [3] if it can be written in the form

$$
P(x)=\sum_{i=1}^{\infty} x_{i}^{*}(x)^{k} y_{i} \quad(x \in E),
$$

where $\left(x_{i}^{*}\right) \subset E^{*}$ and $\left(y_{i}\right) \subset F$ are bounded sequences such that

$$
\sum_{i=1}^{\infty}\left\|x_{i}^{*}\right\|^{k}\left\|y_{i}\right\|<\infty
$$

We denote by $\mathcal{P}_{\mathrm{N}}\left({ }^{k} E, F\right)$ the space of all $k$-homogeneous nuclear polynomials from $E$ into $F$ endowed with the nuclear norm

$$
\|P\|_{\mathrm{N}}:=\inf \sum_{i=1}^{\infty}\left\|x_{i}^{*}\right\|^{k}\left\|y_{i}\right\|,
$$

where the infimum is taken over all bounded sequences $\left(x_{i}^{*}\right) \subset E^{*}$ and $\left(y_{i}\right) \subset F$ which satisfy (1) and (2).

A polynomial $P \in \mathcal{P}\left({ }^{k} E, F\right)$ is Pietsch integral (respectively Grothendieck integral) [3] if there exists a regular countably additive, $F$-valued (respectively $F^{* *}$-valued) Borel measure $\mathscr{G}$ of bounded variation on $B_{E^{*}}$ such that

$$
P(x)=\int_{B_{E^{*}}}\left[x^{*}(x)\right]^{k} d \mathscr{G}\left(x^{*}\right) \quad(x \in E) .
$$

The symbol $\mathcal{P}_{\mathrm{PI}}\left({ }^{k} E, F\right)$ (respectively $\left.\mathcal{P}_{\mathrm{I}}\left({ }^{k} E, F\right)\right)$ denotes the space of all Pietsch integral (respectively integral) $k$-homogeneous polynomials from $E$ into $F$ endowed with the Pietsch integral norm (respectively integral norm)

$$
\left.\|P\|_{\mathrm{PI}}:=\inf |\mathscr{G}|\left(B_{E^{*}}\right) \quad \text { (respectively } \quad\|P\|_{\mathrm{I}}:=\inf |\mathscr{G}|\left(B_{E^{*}}\right)\right),
$$

where $|\mathscr{G}|$ denotes the variation of $\mathscr{G}$ and the infimum is taken over all vector measures $\mathscr{G}$ satisfying the definition. 
It is well known and easy to verify that

$$
\mathcal{P}_{\mathrm{N}}\left({ }^{k} E, F\right) \subseteq \mathcal{P}_{\mathrm{PI}}\left({ }^{k} E, F\right) \subseteq \mathcal{P}_{\mathrm{I}}\left({ }^{k} E, F\right)
$$

with

$$
\|P\| \leq\|P\|_{\mathrm{I}} \leq\|P\|_{\mathrm{PI}} \leq\|P\|_{\mathrm{N}} \quad \text { for every } \quad P \in \mathcal{P}_{\mathrm{N}}\left({ }^{k} E, F\right) .
$$

The space of Pietsch integral operators from $E$ into $F$ is denoted by $\mathcal{P} \mathcal{I}(E, F)$.

We consider in this paper $L_{1}(\mu)$ spaces for finite measures $\mu$, but this covers much more general situations. Indeed, if $v$ is a $\sigma$-finite measure, then there is a probability measure $\mu$ such that $L_{1}(\mu) \equiv L_{1}(v)$ [1, Section 5.1].

A Banach space $F$ has the Radon-Nikodým property if for every finite measure space $(\Omega, \Sigma, \mu)$ and for each $\mu$-continuous vector measure $\mathscr{G}: \Sigma \rightarrow F$ of bounded variation, there exists $g \in L_{1}(\mu, F)$ such that

$$
\mathscr{G}(A)=\int_{A} g d \mu \quad \text { for all } A \in \Sigma .
$$

The space $\ell_{1}$ and the reflexive Banach spaces have the Radon-Nikodým property. The reader is referred to [18, Section VII.6] for many equivalent formulations of the Radon-Nikodým property and to [18, Section VII.7] for a list of spaces with (and without) the Radon-Nikodým property.

We shall need the following essentially known result.

THEOREM 1.2. Given a polynomial $P \in \mathcal{P}\left({ }^{k} E, F\right)$, the following assertions are equivalent:

(a) $P$ is Pietsch integral.

(b) There are a compact Hausdorff space $K$, an into isomorphism

$$
h \in \mathcal{L}(E, C(K)),
$$

a finite nonnegative countably additive, Borel measure $\mu$ on $K$ and an operator

$$
S \in \mathcal{L}\left(L_{1}(K, \mu), F\right)
$$

such that the following diagram is commutative:

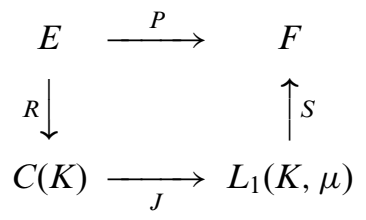

where $J$ is the natural inclusion and $R$ is the polynomial given by

$$
R(x):=[h(x)]^{m} \quad \text { for all } \quad x \in E .
$$

Moreover, if $P$ is Pietsch integral, we have

$$
\|P\|_{\mathrm{PI}}=\inf \|R\|\|S\|\|J\|_{\mathrm{PI}},
$$

where the infimum is taken over all possible factorisations. 
Proof. The equivalence (a) $\Leftrightarrow$ (b) is proved in [16, Theorem 2.3]. Using [17, D5], the equality $\|J\|=\|J\|_{\mathrm{PI}}[\mathbf{1 7}$, Theorem 10.5], and a slight modification of the proof of [16, Theorem 2.3], we obtain the equality of the norms.

As the referee pointed out, Theorem 1.2 can also be obtained from [11, Proposition 2.10].

A similar result holds for integral polynomials, upon replacing $P$ by $j_{F} \circ P$.

THEOREM 1.3. The class of all left $\ell_{1}$-factorable $k$-homogeneous polynomials is a Banach ideal of polynomials, endowed with the norm $\gamma_{\ell_{1}}^{\text {left. }}$

Proof. By [23, Criterion 1.4], which is also valid for vector-valued polynomials, we need to show that given a sequence $\left(P_{n}\right) \subset \mathcal{P}\left({ }^{k} E, F\right)$ of left $\ell_{1}$-factorable polynomials so that

$$
\sum_{n=1}^{\infty} \gamma_{\ell_{1}}^{\text {left }}\left(P_{n}\right)<+\infty
$$

$P:=\sum_{n=1}^{\infty} P_{n}$ is left $\ell_{1}$-factorable and

$$
\gamma_{\ell_{1}}^{\text {left }}(P) \leq \sum_{n=1}^{\infty} \gamma_{\ell_{1}}^{\text {left }}\left(P_{n}\right)
$$

Since $\left\|P_{n}\right\| \leq \gamma_{\ell_{1}}^{\text {left }}\left(P_{n}\right)$ for all $n \in \mathbb{N}$, the series $\sum_{n=1}^{\infty} P_{n}$ is absolutely convergent in the Banach space $\mathcal{P}\left({ }^{k} E, F\right)$ and so it is convergent. Let $P:=\sum_{n=1}^{\infty} P_{n}$. For each $n \in \mathbb{N}$ and $\epsilon>0$, there are a polynomial $Q_{n} \in \mathcal{P}\left({ }^{k} E, \ell_{1}\right)$ and an operator $T_{n} \in \mathcal{L}\left(\ell_{1}, F\right)$ so that $P_{n}=T_{n} \circ Q_{n}$, with $\left\|T_{n}\right\| \leq 1$ and $\left\|Q_{n}\right\| \leq \gamma_{\ell_{1}}^{\text {left }}\left(P_{n}\right)+\epsilon 2^{-n}$. Define the polynomial

$$
Q: E \longrightarrow\left(\sum_{n=1}^{\infty} \ell_{1}\right)_{1}
$$

by

$$
Q(x):=\left(Q_{n}(x)\right)_{n=1}^{\infty} \quad \text { for } x \in E .
$$

Clearly, $Q$ is well defined. Let

$$
T:\left(\sum_{n=1}^{\infty} \ell_{1}\right)_{1} \longrightarrow F
$$

be the operator given by

$$
T\left(\left(x_{n}\right)_{n=1}^{\infty}\right):=\sum_{n=1}^{\infty} T_{n}\left(x_{n}\right), \quad \text { for } x_{n} \in \ell_{1}(n \in \mathbb{N}) .
$$

Since

$$
\left\|T\left(\left(x_{n}\right)_{n=1}^{\infty}\right)\right\| \leq \sum_{n=1}^{\infty}\left\|T_{n}\left(x_{n}\right)\right\| \leq \sum_{n=1}^{\infty}\left\|x_{n}\right\|=\left\|\left(x_{n}\right)_{n=1}^{\infty}\right\|,
$$

$T$ is well defined, with $\|T\| \leq 1$. 
On the other hand, we have

$$
T \circ Q(x)=\sum_{n=1}^{\infty} T_{n}\left(Q_{n}(x)\right)=\sum_{n=1}^{\infty} P_{n}(x)=P(x) \quad(x \in E) .
$$

Recalling that $\left(\sum_{n=1}^{\infty} \ell_{1}\right)_{1}$ is isometrically isomorphic to $\ell_{1}$ [35, Examples II.B.22], we have that $P$ is left $\ell_{1}$-factorable. Moreover,

$$
\gamma_{\ell_{1}}^{\text {left }}(P) \leq\|T\|\|Q\| \leq\|Q\| \leq \sum_{n=1}^{\infty}\left\|Q_{n}\right\| \leq \sum_{n=1}^{\infty} \gamma_{\ell_{1}}^{\text {left }}\left(P_{n}\right)+\epsilon .
$$

Since $\epsilon>0$ is arbitrary, we obtain the desired result (4). It is easy to see that the other conditions of [23, Criterion 1.4] are fulfilled.

2. Relationship with other ideals of polynomials. In this section, we show that every nuclear polynomial factors through a nuclear polynomial into $\ell_{1}$, and we obtain norm estimates of the factors. We give a characterisation of the Radon-Nikodým property in terms of left $\ell_{1}$-factorisation of polynomials, extending Theorem 1.1 to the polynomial setting. Finally, we characterise $\mathscr{L}_{1}$-spaces by the left compact $\ell_{1}$ factorisation of compact polynomials.

The ideal of nuclear polynomials is the smallest Banach ideal of polynomials. This is proved in [22, Proposition 1.7] in the scalar-valued case, but the proof is also valid in the vector-valued setting. Hence, every nuclear polynomial $P$ is left $\ell_{1}$-factorable and

$$
\gamma_{\ell_{1}}^{\text {left }}(P) \leq\|P\|_{\mathrm{N}}
$$

We shall however give a theorem on left nuclear $\ell_{1}$-factorisation of nuclear polynomials in order to obtain norm equalities which are interesting in their own right. We shall see that every nuclear polynomial factors through a 'standard' nuclear polynomial that we consider in the next proposition whose proof is simple.

Proposition 2.1. Given $\lambda=\left(\lambda_{n}\right) \in \ell_{1}$, the polynomial $M_{\lambda} \in \mathcal{P}\left({ }^{k} \ell_{\infty}, \ell_{1}\right)$ defined by

$$
M_{\lambda}(x):=\left(\lambda_{n} x_{n}^{k}\right)_{n=1}^{\infty}, \quad \text { for } x=\left(x_{n}\right)_{n=1}^{\infty} \in \ell_{\infty},
$$

is nuclear and

$$
\left\|M_{\lambda}\right\|=\left\|M_{\lambda}\right\|_{\mathrm{N}}=\|\lambda\| .
$$

This result is also valid if the domain space is $c_{0}$ instead of $\ell_{\infty}$.

The factorisations of the next theorem are proved in [13, Proposition 6]. Here we add the equalities of the norms. We first give a (probably well-known) preparatory lemma. Its proof is easy, using [30, Exercise 3.12].

LEMmA 2.2. Given $\lambda=\left(\lambda_{n}\right) \in \ell_{1}$ and $0<\epsilon<2$, there are $\left(\alpha_{n}\right)_{n=1}^{\infty} \in c_{0}$ with $0 \leq$ $\alpha_{n} \leq 1$ for all $n \in \mathbb{N}$ and $\tau:=\left(\tau_{n}\right) \in \ell_{1}$ such that $\lambda_{n}=\alpha_{n} \tau_{n}$, with

$$
\|\tau\| \leq\|\lambda\|+\epsilon \text {. }
$$


TheOrem 2.3. Let $P \in \mathcal{P}\left({ }^{k} E, F\right)$ be a polynomial. The following assertions are equivalent:

(a) $P$ is nuclear.

(b) There are operators $U \in \mathcal{L}\left(E, \ell_{\infty}\right)$ and $V \in \mathcal{L}\left(\ell_{1}, F\right)$ and a polynomial $M_{\lambda} \in$ $\mathcal{P}\left({ }^{k} \ell_{\infty}, \ell_{1}\right)$ of the form $M_{\lambda}(z)=\left(\lambda_{n} z_{n}^{k}\right)_{n=1}^{\infty}$, where $\lambda=\left(\lambda_{n}\right) \in \ell_{1}$ and $z=\left(z_{n}\right) \in \ell_{\infty}$, such that the following diagram commutes:

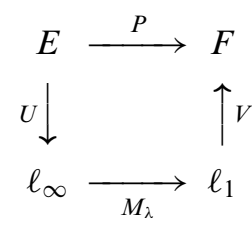

(c) There are compact operators $T \in \mathcal{K}\left(E, c_{0}\right)$ and $S \in \mathcal{K}\left(\ell_{1}, F\right)$ and a polynomial $M_{\tau}^{\prime} \in \mathcal{P}\left({ }^{k} c_{0}, \ell_{1}\right)$ of the form $M_{\tau}^{\prime}(y)=\left(\tau_{n} y_{n}^{k}\right)_{n=1}^{\infty}$, where $\tau:=\left(\tau_{n}\right) \in \ell_{1}$ and $y=\left(y_{n}\right) \in c_{0}$, such that the following diagram commutes:

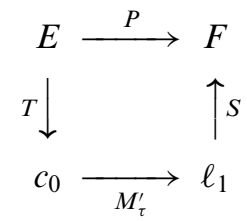

Moreover, if $P$ is nuclear, we have

$$
\|P\|_{\mathrm{N}}=\inf \|U\|^{k}\|\lambda\|\|V\|=\inf \|T\|^{k}\|\tau\|\|S\|,
$$

where the infimum is taken over all factorisations as in (b) and (c).

Proof. (a) $\Rightarrow$ (b). Assume $P \in \mathcal{P}_{\mathrm{N}}\left({ }^{k} E, F\right)$. For every $\epsilon>0$, there are bounded sequences $\left(\phi_{n}\right) \subset E^{*}$ and $\left(y_{n}\right) \subset F$ such that

$$
P(x)=\sum_{n=1}^{\infty} \phi_{n}(x)^{k} y_{n} \quad \text { for all } x \in E, \text { with } \quad \sum_{n=1}^{\infty}\left\|\phi_{n}\right\|^{k}\left\|y_{n}\right\|<\|P\|_{\mathrm{N}}+\epsilon .
$$

Define $U \in \mathcal{L}\left(E, \ell_{\infty}\right), V \in \mathcal{L}\left(\ell_{1}, F\right)$ and $\lambda=\left(\lambda_{n}\right) \in \ell_{1}$ by

$$
\begin{aligned}
U(x) & :=\left(\frac{\phi_{n}(x)}{\left\|\phi_{n}\right\|}\right)_{n=1}^{\infty} \in \ell_{\infty} \quad(x \in E), \\
\lambda_{n} & :=\left\|\phi_{n}\right\|^{k}\left\|y_{n}\right\| \quad(n \in \mathbb{N}), \\
V\left(e_{n}\right) & :=\frac{y_{n}}{\left\|y_{n}\right\|} \in F \quad(n \in \mathbb{N}) .
\end{aligned}
$$

Then, we easily obtain

$$
V \circ M_{\lambda} \circ U(x)=P(x) \quad(x \in E)
$$

so the diagram of (b) is commutative. Moreover, $\|V\|=\|U\|=1$ and

$$
\|P\|_{\mathrm{N}}=\left\|V \circ M_{\lambda} \circ U\right\|_{\mathrm{N}} \leq\|V\|\|\lambda\|\|U\|^{k}=\|\lambda\|=\sum_{n=1}^{\infty}\left\|\phi_{n}\right\|^{k}\left\|y_{n}\right\|<\|P\|_{\mathrm{N}}+\epsilon,
$$


and we conclude that

$$
\|P\|_{\mathrm{N}}=\inf \|V\|\|\lambda\|\|U\|^{k} .
$$

(a) $\Rightarrow$ (c). Let $P \in \mathcal{P}_{\mathrm{N}}\left({ }^{k} E, F\right)$. Given $0<\epsilon<2$, we can find a factorisation as in (b) with $\|U\|=\|V\|=1$ and

$$
\|P\|_{\mathrm{N}} \leq\|\lambda\|<\|P\|_{\mathrm{N}}+\frac{\epsilon}{2} .
$$

Then, by Lemma 2.2, there are $\left(\alpha_{n}\right)_{n=1}^{\infty} \in c_{0}$ with $0 \leq \alpha_{n} \leq 1$ for all $n \in \mathbb{N}$ and $\tau:=$ $\left(\tau_{n}\right) \in \ell_{1}$ such that $\lambda_{n}=\alpha_{n} \tau_{n}$, with

$$
\|\tau\| \leq\|\lambda\|+\frac{\epsilon}{2}
$$

Define operators $B \in \mathcal{L}\left(\ell_{\infty}, c_{0}\right)$ and $A \in \mathcal{L}\left(\ell_{1}, \ell_{1}\right)$ and a polynomial $M_{\tau}^{\prime} \in \mathcal{P}\left({ }^{k} c_{0}, \ell_{1}\right)$ by

$$
\begin{aligned}
B(z): & =\left(\alpha_{n}^{1 /(2 k)} z_{n}\right)_{n=1}^{\infty}, \quad \text { for } z=\left(z_{n}\right) \in \ell_{\infty}, \\
A(\omega): & =\left(\alpha_{n}^{1 / 2} \omega_{n}\right)_{n=1}^{\infty}, \quad \text { for } \omega=\left(\omega_{n}\right) \in \ell_{1}, \\
M_{\tau}^{\prime}(y): & =\left(\tau_{n} y_{n}^{k}\right)_{n=1}^{\infty}, \quad \text { for } y=\left(y_{n}\right) \in c_{0} .
\end{aligned}
$$

Then, $A$ and $B$ are compact with norm $\leq 1$ and

$$
M_{\lambda}=A \circ M_{\tau}^{\prime} \circ B
$$

so the following diagram is commutative:

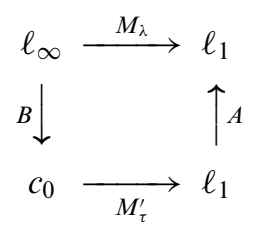

Let $T:=B \circ U$ and $S:=V \circ A$. Then

$$
P=V \circ M_{\lambda} \circ U=V \circ A \circ M_{\tau}^{\prime} \circ B \circ U=S \circ M_{\tau}^{\prime} \circ T
$$

so

$$
\begin{aligned}
\|P\|_{\mathrm{N}} & \leq\|S\|\left\|M_{\tau}^{\prime}\right\|_{\mathrm{N}}\|T\|^{k} \\
& =\|S\|\|\tau\|\|T\|^{k}, \\
& \left.\leq\|A\|\|\tau\|\|B\|^{k} \quad \text { (since }\|U\|=\|V\|=1\right) \\
& \leq\|\lambda\|+\frac{\epsilon}{2}, \\
& <\|P\|_{\mathrm{N}}+\epsilon
\end{aligned}
$$

and therefore

$$
\|P\|_{\mathrm{N}}=\inf \|S\|\|\tau\|\|T\|^{k}
$$


(b) $\Rightarrow$ (a) and (c) $\Rightarrow$ (a). It is enough to use Proposition 2.1 and the comment after it and the ideal property.

Corollary 2.4. For every $P \in \mathcal{P}_{\mathrm{N}}\left({ }^{k} E, F\right)$, there are a polynomial $Q \in \mathcal{P}_{\mathrm{N}}\left({ }^{k} E, \ell_{1}\right)$ and an operator $T \in \mathcal{L}\left(\ell_{1}, F\right)$ such that $P=T \circ Q$ and

$$
\|P\|_{\mathrm{N}}=\inf \|Q\|_{\mathrm{N}}\|T\|,
$$

where the infimum is taken over all possible factorisations.

Proof. By Theorem 2.3, we have a commutative diagram

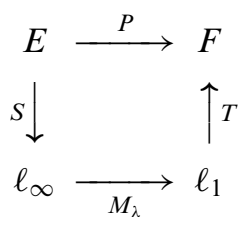

and

$$
\|P\|_{\mathrm{N}}=\left\|T \circ M_{\lambda} \circ S\right\|_{\mathrm{N}} \leq\|T\|\left\|M_{\lambda} \circ S\right\|_{\mathrm{N}} \leq\|T\|\|\lambda\|\|S\|^{k} .
$$

Given $\epsilon>0$, we can choose $S, \lambda$ and $T$ so that

$$
\|T\|\|\lambda\|\|S\|^{k}<\|P\|_{\mathrm{N}}+\epsilon .
$$

So, letting $Q:=M_{\lambda} \circ S \in \mathcal{P}_{\mathrm{N}}\left({ }^{k} E, \ell_{1}\right)$, we have

$$
\|P\|_{\mathrm{N}}=\inf \|Q\|_{\mathrm{N}}\|T\|,
$$

and the proof is finished.

Using Theorem 2.3(c) we can assume $T$ in Corollary 2.4 to be compact.

Recall [7, p. 168] that for each $i \in \mathbb{N}$, there are operators

$$
\pi_{i}: \otimes_{\pi, s}^{i+1} E \longrightarrow \otimes_{\pi, s}^{i} E \quad \text { and } \quad j_{i}: \otimes_{\pi, s}^{i} E \longrightarrow \otimes_{\pi, s}^{i+1} E
$$

such that $\pi_{i} \circ j_{i}$ is the identity map on $\otimes_{\pi, s}^{i} E$.

Observe that if a polynomial $P \in \mathcal{P}\left({ }^{k} E, F\right)$ is left $\ell_{1}$-factorable in the form $P=L \circ Q$, then its linearisation $\bar{P} \in \mathcal{L}\left(\otimes_{\pi, s}^{k} E, F\right)$ is $\ell_{1}$-factorable in the form $\bar{P}=L \circ \bar{Q}$.

Proposition 2.5. Let $E$ and $F$ be Banach spaces, and let $k \in \mathbb{N}$. Assume that every polynomial $P \in \mathcal{P}\left({ }^{k} E, F\right)$ is left $\ell_{1}$-factorable. Then every operator $T \in \mathcal{L}(E, F)$ is $\ell_{1}$ factorable.

Proof. Let $T \in \mathcal{L}(E, F)$ be given. Consider the polynomial

$$
P:=T \circ \pi_{1} \circ \ldots \circ \pi_{k-1} \circ \delta_{k}: E \longrightarrow F .
$$

By the hypothesis, $P$ is left $\ell_{1}$-factorable. By the above comment, its linearisation

$$
\bar{P}=T \circ \pi_{1} \circ \ldots \circ \pi_{k-1}: \otimes_{\pi, s}^{k} E \longrightarrow F
$$


is $\ell_{1}$-factorable. It follows that the operator

$$
T=T \circ \pi_{1} \circ \cdots \circ \pi_{k-1} \circ j_{k-1} \circ \cdots \circ j_{1} \in \mathcal{L}(E, F)
$$

is also $\ell_{1}$-factorable.

REMARK 2.6. In Proposition 2.5, from the fact that every $k$-homogeneous polynomial between $E$ and $F$ satisfies a property, we deduce that every operator between $E$ and $F$ satisfies the same property. This is also done in the proof of Theorem 2.9, part (b) $\Rightarrow$ (d), and in Proposition 3.1.

A general procedure (not using tensor products) to pass from properties of ideals of polynomials to properties of operator ideals and vice versa has been developed in [8] and [10, Proposition 1.6].

THEOREM 2.7. Given a Banach space $F$, the following assertions are equivalent:

(a) $F$ has the Radon-Nikodým property;

(b) for every finite measure space $(\Omega, \Sigma, \mu)$ and for every $k \in \mathbb{N}$, every polynomial $P \in \mathcal{P}\left({ }^{k} L_{1}(\mu), F\right)$ is left $\ell_{1}$-factorable;

(c) for every finite measure space $(\Omega, \Sigma, \mu)$, there is an integer $k \in \mathbb{N}$ such that every polynomial $P \in \mathcal{P}\left({ }^{k} L_{1}(\mu), F\right)$ is left $\ell_{1}$-factorable.

Proof. (a) $\Rightarrow$ (b). Let $P \in \mathcal{P}\left({ }^{k} L_{1}(\mu), F\right)$ be a polynomial. Since $\otimes_{\pi}^{k} L_{1}(\mu)$ is isometrically isomorphic to $L_{1}(v)$ for some finite measure $v$ [33, Exercise 2.8], by Theorem 1.1, there are operators $S \in \mathcal{L}\left(\otimes_{\pi}^{k} L_{1}(\mu), \ell_{1}\right)$ and $L \in \mathcal{L}\left(\ell_{1}, F\right)$ such that

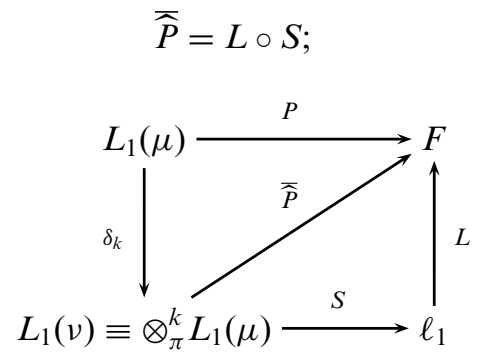

Then, $P=L \circ Q$, with $Q:=S \circ \delta_{k} \in \mathcal{P}\left({ }^{k} L_{1}(\mu), \ell_{1}\right)$.

(b) $\Rightarrow$ (c) is obvious.

(c) $\Rightarrow$ (a). By Proposition 2.5, every operator $T \in \mathcal{L}\left(L_{1}(\mu), F\right)$ is $\ell_{1}$-factorable. By the Lewis-Stegall theorem (Theorem 1.1), $F$ has the Radon-Nikodým property.

We do not know if the equality $\|P\|=\inf \|Q\|\|L\|$ holds, where the infimum should be taken over all possible factorisations. It seems to us that in order to prove such an equality, we would need to introduce the concept of representable polynomials, which is beyond the scope of this paper.

REMARK 2.8. If $P \in \mathcal{P}\left({ }^{k} L_{1}(\mu), F\right)$ is a weakly compact polynomial, there are a reflexive Banach space $G$, a polynomial $Q \in \mathcal{P}\left({ }^{k} L_{1}(\mu), G\right)$ and an operator $U \in \mathcal{L}(G, F)$ such that $P=U \circ Q[32$, Theorem 3.7]. Since $G$ has the Radon-Nikodým property, Theorem 2.7 implies that $P$ is left $\ell_{1}$-factorable.

We now study the left $\ell_{1}$-factorisation of compact polynomials on $\mathscr{L}_{1}$-spaces. The definition and basic properties of $\mathscr{L}_{p}$-spaces may be seen in $[27,28]$. We say that an 
operator $T \in \mathcal{K}(E, F)$ factors compactly through $\ell_{1}$ if there are operators $S \in \mathcal{K}\left(E, \ell_{1}\right)$ and $L \in \mathcal{K}\left(\ell_{1}, F\right)$ such that $T=L \circ S$.

THEOREM 2.9. Given a Banach space E, the following assertions are equivalent:

(a) $E$ is an $\mathscr{L}_{1}$-space;

(b) for every (some) $k \in \mathbb{N}$ and every Banach space $F$, for every polynomial $P \in$ $\mathcal{P}_{\mathrm{K}}\left({ }^{k} E, F\right)$, there are a polynomial $Q \in \mathcal{P}_{\mathrm{K}}\left({ }^{k} E, \ell_{1}\right)$ and an operator $L \in \mathcal{K}\left(\ell_{1}, F\right)$ such that $P=L \circ Q$;

(c) for every (some) $k \in \mathbb{N}$ and every Banach space $F$, for every polynomial $P \in$ $\mathcal{P}_{\mathrm{wb}}\left({ }^{k} E, F\right)$, there are a polynomial $Q \in \mathcal{P}_{\mathrm{wb}}\left({ }^{k} E, \ell_{1}\right)$ and an operator $L \in \mathcal{K}\left(\ell_{1}, F\right)$ such that $P=L \circ Q$;

(d) for every Banach space $F$, every operator $T \in \mathcal{K}(E, F)$ factors compactly through $\ell_{1}$.

Proof. (a) $\Rightarrow$ (b). Suppose that $E$ is an $\mathscr{L}_{1}$-space. Let $P \in \mathcal{P}_{\mathrm{K}}\left({ }^{k} E, F\right)$ be a compact polynomial. Then the linearisation $\bar{P}$ is compact [31, Lemma 4.1]. By [20, Corollary 3.3], there are a Banach space $G$ and operators $A \in \mathcal{K}\left(\otimes_{\pi, s}^{k} E, G\right)$ and $B \in \mathcal{K}(G, F)$ such that $\bar{P}=B \circ A$ :

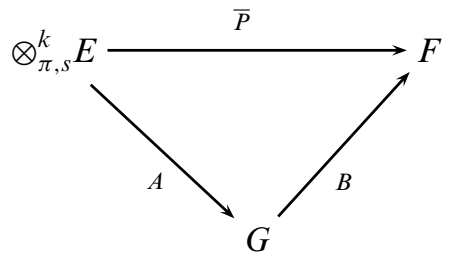

The range of $A^{*}$ is in $\mathcal{P}\left({ }^{k} E\right) \simeq\left(\otimes_{\pi, s}^{k} E\right)^{*}$ which is an $\mathscr{L}_{\infty}$-space [14, p. 233]. By [26, Theorem 2], $A^{*}$ factors compactly through $c_{0}$; so there are operators $U \in \mathcal{K}\left(G^{*}, c_{0}\right)$ and $V \in \mathcal{K}\left(c_{0}, \mathcal{P}\left({ }^{k} E\right)\right)$ such that $A^{*}=V \circ U$ :

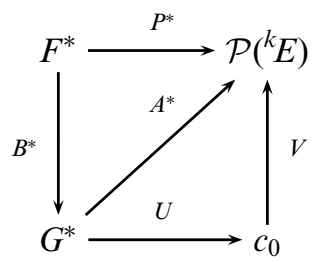

Taking adjoints, we have that $P^{* *}$ factors compactly through $\ell_{1}$. Note that the ranges of $P^{* *}$ and $B^{* *}$ are contained in $F$ :

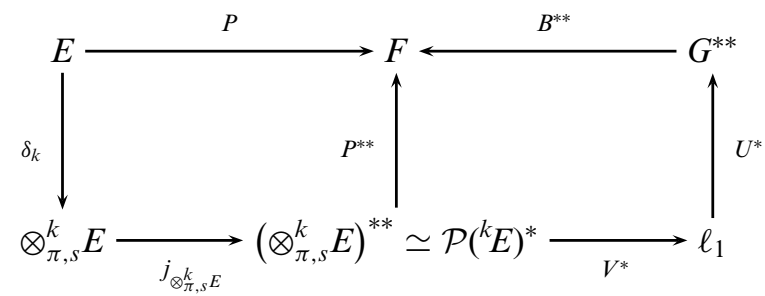

Since the above diagram is commutative, letting $Q:=V^{*} \circ j_{\otimes_{\pi, s}^{k} E} \circ \delta_{k} \in \mathcal{P}_{\mathrm{K}}\left({ }^{k} E, \ell_{1}\right)$ and $L:=B^{* *} \circ U^{*} \in \mathcal{K}\left(\ell_{1}, F\right)$, the result is proved.

(b) $\Rightarrow$ (d). Suppose (b) holds for some $k \in \mathbb{N}$. A simple modification of the proof of Proposition 2.5 allows us to obtain (d).

(d) $\Rightarrow$ (c). Let $P \in \mathcal{P}_{\text {wb }}\left({ }^{k} E, F\right)$ be a polynomial whose restrictions to bounded subsets are weakly continuous. By [25, Corollary 15], there are a Banach space $G$, an 
operator $S \in \mathcal{K}(E, G)$ and a polynomial $R \in \mathcal{P}_{\text {wb }}\left({ }^{k} G, F\right)$ such that $P=R \circ S$. By (d), there are operators $A \in \mathcal{K}\left(E, \ell_{1}\right)$ and $B \in \mathcal{K}\left(\ell_{1}, G\right)$ such that $S=B \circ A$ :

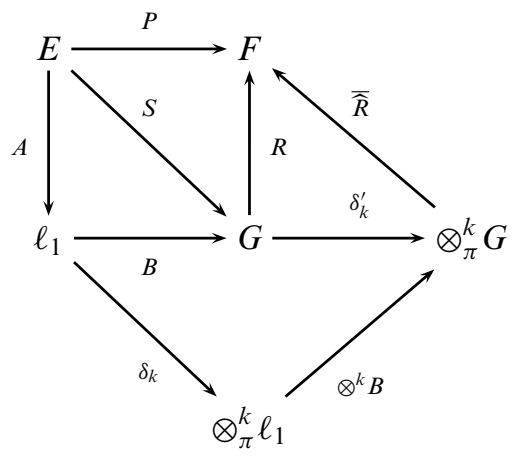

Let $\delta_{k}: \ell_{1} \rightarrow \otimes_{\pi}^{k} \ell_{1}$ and $\delta_{k}^{\prime}: G \rightarrow \otimes_{\pi}^{k} G$ be the canonical polynomials. Let $Q:=$ $\delta_{k} \circ A \in \mathcal{P}_{\mathrm{wb}}\left({ }^{k} E, \otimes_{\pi}^{k} \ell_{1}\right)$ and let $L:=\overline{\widehat{R}} \circ\left(\otimes^{k} B\right) \in \mathcal{K}\left(\otimes_{\pi}^{k} \ell_{1}, F\right)(L$ is compact since $\widehat{\widehat{R}}$ is compact). Then,

$$
P=R \circ S=R \circ B \circ A=\widehat{\widehat{R}} \circ \delta_{k}^{\prime} \circ B \circ A=\widehat{\widehat{R}} \circ\left(\otimes^{k} B\right) \circ \delta_{k} \circ A=L \circ Q .
$$

Since $\otimes_{\pi}^{k} \ell_{1}$ is isometrically isomorphic to $\ell_{1}$ [33, Exercise 2.6], the result is proved.

(c) $\Rightarrow$ (a). Let $P \in \mathcal{P}_{\mathrm{wb}}\left({ }^{k} E, F\right)$ be a polynomial whose restrictions to bounded subsets are weakly continuous, and let $S \in \mathcal{L}(G, F)$ be a surjective operator. By (c), there are a polynomial $R \in \mathcal{P}_{\mathrm{wb}}\left({ }^{k} E, \ell_{1}\right)$ and an operator $L \in \mathcal{K}\left(\ell_{1}, F\right)$ such that $P=L \circ R$ :

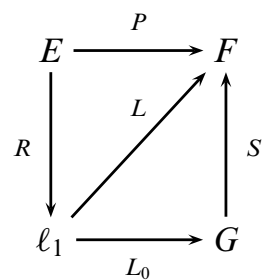

Since $\ell_{1}$ is an $\mathscr{L}_{1}$-space, $L$ has a compact lifting $L_{0} \in \mathcal{K}\left(\ell_{1}, G\right)$ such that $L=S \circ L_{0}$ [28, Theorem 4.2]. Let $Q:=L_{0} \circ R \in \mathcal{P}_{\mathrm{wb}}\left({ }^{k} E, G\right)$, which is a lifting of $P$, since

$$
P=L \circ R=S \circ L_{0} \circ R=S \circ Q .
$$

By [14, Theorem 7], $E$ is an $\mathscr{L}_{1}$-space.

3. Left integral $\ell_{1}$-factorisation property. In this section, we introduce the left integral $\ell_{1}$-factorisation property of Pietsch integral polynomials. We prove that it characterises the Radon-Nikodým property and show that it plays to some extent the role of nuclear operators with range in spaces with the Radon-Nikodým property. As an application, we give a result on composition of polynomials and operators.

We say that a polynomial $P \in \mathcal{P}_{\mathrm{PI}}\left({ }^{k} E, F\right)$ has the left integral $\ell_{1}$-factorisation property if there are a polynomial $Q \in \mathcal{P}_{\mathrm{PI}}\left({ }^{k} E, \ell_{1}\right)$ and an operator $L \in \mathcal{L}\left(\ell_{1}, F\right)$ such that $P=L \circ Q$.

The operators $\pi_{i}$ and $j_{i}$ used in the proof of Proposition 2.5 are also continuous when the tensor products are endowed with the injective topology $[4,3.5]$. This allows us to obtain the next result. 
Proposition 3.1. Let $E$ and $F$ be Banach spaces, and let $k \in \mathbb{N}$. Assume that every polynomial $\left.P \in \mathcal{P}_{\mathrm{PI}}{ }^{k} E, F\right)$ has the left integral $\ell_{1}$-factorisation property. Then, for every operator $T \in \mathcal{P} \mathcal{I}(E, F)$, there are operators $S \in \mathcal{P} \mathcal{I}\left(E, \ell_{1}\right)$ and $L \in \mathcal{L}\left(\ell_{1}, F\right)$ such that $T=L \circ S$.

Proof. Let $T \in \mathcal{P} \mathcal{I}(E, F)$ be given. Define a polynomial $P$ as the composition of the following mappings:

$$
E \stackrel{\delta_{k}}{\longrightarrow} \otimes_{\epsilon, s}^{k} E \stackrel{\pi_{k-1}}{\longrightarrow} \otimes_{\epsilon, s}^{k-1} E \stackrel{\pi_{k-2}}{\longrightarrow} \cdots \stackrel{\pi_{1}}{\longrightarrow} E \stackrel{T}{\longrightarrow} F .
$$

Since its linearisation $\bar{P}$, given by the composition

$$
\otimes_{\epsilon, s}^{k} E \stackrel{\pi_{k-1}}{\longrightarrow} \otimes_{\epsilon, s}^{k-1} E \stackrel{\pi_{k-2}}{\longrightarrow} \cdots \stackrel{\pi_{1}}{\longrightarrow} E \stackrel{T}{\longrightarrow} F,
$$

is continuous and Pietsch integral, $P$ is also Pietsch integral [34, Corollary 2.8]. So there are a polynomial $Q \in \mathcal{P}_{\mathrm{PI}}\left({ }^{k} E, \ell_{1}\right)$ and an operator $L \in \mathcal{L}\left(\ell_{1}, F\right)$ such that $P=L \circ Q$ :

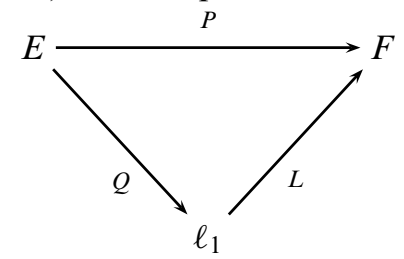

Hence, the operator $\bar{P}$, defined on $\otimes_{\epsilon, s}^{k} E$, is $\ell_{1}$-factorable:

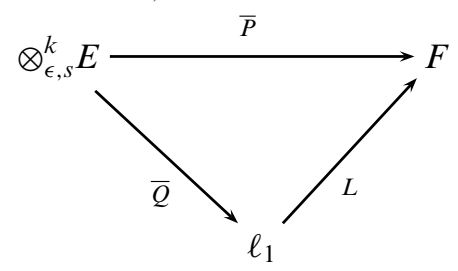

with $\bar{Q} \in \mathcal{P} \mathcal{I}\left(\otimes_{\epsilon, S}^{k} E, \ell_{1}\right)$ again by [34, Corollary 2.8]. Since

$$
T=T \circ \pi_{1} \circ \cdots \circ \pi_{k-1} \circ j_{k-1} \circ \cdots \circ j_{1}=\bar{P} \circ j_{k-1} \circ \cdots \circ j_{1},
$$

$T$ is also $\ell_{1}$-factorable through a Pietsch integral operator.

THEOREM 3.2. Given a Banach space $F$, the following assertions are equivalent:

(a) $F$ has the Radon-Nikodým property;

(b) for every (some) $k \in \mathbb{N}$ and for every Banach space $E$, every polynomial $P \in$ $\mathcal{P}_{\mathrm{PI}}\left({ }^{k} E, F\right)$ has the left integral $\ell_{1}$-factorisation property;

(c) for every (some) $k \in \mathbb{N}$, every polynomial $P \in \mathcal{P}_{\mathrm{PI}}\left({ }^{k} C[0,1], F\right)$ has the left integral $\ell_{1}$-factorisation property;

(d) for every Banach space $E$ and every operator $T \in \mathcal{P} \mathcal{I}(E, F)$, there are operators $S \in \mathcal{P} \mathcal{I}\left(E, \ell_{1}\right)$ and $L \in \mathcal{L}\left(\ell_{1}, F\right)$ such that $T=L \circ S$;

(e) for every operator $T \in \mathcal{P} \mathcal{I}(C[0,1], F)$, there are operators $S \in \mathcal{P} \mathcal{I}\left(C[0,1], \ell_{1}\right)$ and $L \in \mathcal{L}\left(\ell_{1}, F\right)$ such that $T=L \circ S$;

(f) every operator $T \in \mathcal{P} \mathcal{I}(C[0,1], F)$ is nuclear.

Moreover, if $F$ has the Radon-Nikodým property and $P \in \mathcal{P}_{\mathrm{PI}}\left({ }^{k} E, F\right)$, then

$$
\|P\|_{\mathrm{PI}}=\inf \|Q\|_{\mathrm{PI}}\|L\|,
$$


where the infimum is taken over all factorisations as in (b). And if $T \in \mathcal{P} \mathcal{I}(E, F)$, then

$$
\|T\|_{\mathrm{PI}}=\inf \|S\|_{\mathrm{PI}}\|L\|,
$$

where the infimum is taken over all factorisations as in (d).

Proof. (a) $\Rightarrow$ (b). Let $P \in \mathcal{P}_{\mathrm{PI}}\left({ }^{k} E, F\right)$. By Theorem 1.2, given $\epsilon>0$, there is a factorisation $P=S \circ J \circ R$ (see the diagram below) with

$$
\|P\|_{\mathrm{PI}} \leq\|R\|\|S\|\|J\|_{\mathrm{PI}}<\|P\|_{\mathrm{PI}}+\frac{\epsilon}{2}
$$

Since $F$ has the Radon-Nikodým property, applying Theorem 1.1, we can find operators $A \in \mathcal{L}\left(L_{1}(K, \mu), \ell_{1}\right)$ and $L \in \mathcal{L}\left(\ell_{1}, F\right)$ so that $S=L \circ A$ :

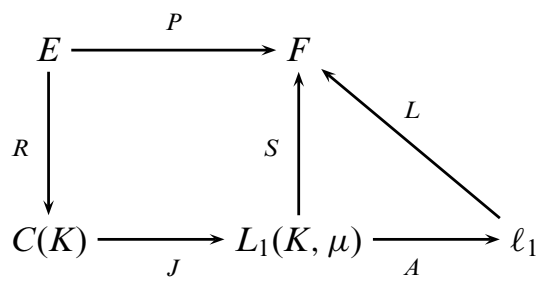

Moreover, we can assume that

$$
\|S\| \leq\|A\|\|L\|<\|S\|+\frac{\epsilon}{2\|R\|\|J\|_{\mathrm{PI}}} .
$$

Let $Q:=A \circ J \circ R$. By Theorem 1.2, $Q$ is a Pietsch integral polynomial. Then, by the ideal property,

$$
\begin{aligned}
\|P\|_{\mathrm{PI}} & \leq\|Q\|_{\mathrm{PI}}\|L\| \\
& \leq\|R\|\|A \circ J\|_{\mathrm{PI}}\|L\| \quad(\text { see Remark 3.3) } \\
& \leq\|A\|\|J\|_{\mathrm{PI}}\|R\|\|L\| \\
& <\|R\|\|J\|_{\mathrm{PI}}\left(\|S\|+\frac{\epsilon}{2\|R\|\|J\|_{\mathrm{PI}}}\right) \\
& =\|R\|\|J\|_{\mathrm{PI}}\|S\|+\frac{\epsilon}{2} \\
& <\|P\|_{\mathrm{PI}}+\epsilon
\end{aligned}
$$

Hence,

$$
\|P\|_{\mathrm{PI}}=\inf \|Q\|_{\mathrm{PI}}\|L\|,
$$

where the infimum is taken over all possible factorisations of the form $P=L \circ Q$, with $Q$ a Pietsch integral polynomial into $\ell_{1}$.

(b) $\Rightarrow$ (c) and (d) $\Rightarrow$ (e) are obvious.

(b) $\Rightarrow$ (d) and (c) $\Rightarrow$ (e) by Proposition 3.1.

(e) $\Rightarrow$ (f). Since $S$ is Pietsch integral and $\ell_{1}$ has the Radon-Nikodým property, $S$ is nuclear [18, Corollary VI.4.5]. Therefore, $T=L \circ S$ is nuclear.

(f) $\Rightarrow$ (a). Let $T \in \mathcal{L}(C[0,1], F)$ be an absolutely summing operator. By [17, Proposition D6], $T$ is Pietsch integral. By (f), $T$ is nuclear. By [18, Corollary VI.4.6], $F$ has the Radon-Nikodým property. 
The argument used in the proof of (a) $\Rightarrow$ (b) to obtain equality (7) is also valid in the linear case $(k=1)$. Hence, if $T \in \mathcal{P} \mathcal{I}(E, F)$, we have

$$
\|T\|_{\text {PI }}=\inf \|S\|_{\text {PI }}\|L\|,
$$

where the infimum is taken over all factorisations as in (d), and this finishes the proof.

REMARK 3.3. In the formulas of (6), we use the inequality

$$
\|Q\|_{\mathrm{PI}} \leq\|R\|\|A \circ J\|_{\mathrm{PI}}
$$

which is an easy consequence of known results. Indeed, if $\bar{Q}$ and $\bar{R}$ are the linearisations of $Q$ and $R$ defined on the $k$-fold symmetric tensor product of $E$ endowed with the symmetric injective tensor norm, then we have

$$
\begin{aligned}
\|Q\|_{\mathrm{PI}} & =\|\bar{Q}\|_{\mathrm{PI}} \quad[\mathbf{1 1}, \text { Proposition 2.10], } \\
& =\|A \circ J \circ \bar{R}\|_{\mathrm{PI}} \quad[\mathbf{1 6}, \text { Lemma 2.2], } \\
& \leq\|A \circ J\|_{\mathrm{PI}}\|\bar{R}\|, \quad \\
& =\|A \circ J\|_{\mathrm{PI}}\|R\| \quad[\mathbf{1 6}, \text { Lemma 2.2]. }
\end{aligned}
$$

REMARKS 3.4. (a) If $F$ has the Radon-Nikodým property, since the operator $J$ in diagram (5) is Pietsch integral, then $S \circ J$ is nuclear [18, Corollary VI.4.5] with

$$
\|S \circ J\|_{\mathrm{N}}=\|S \circ J\|_{\mathrm{PI}} \leq\|S\|\|J\|_{\mathrm{PI}} .
$$

Hence, $P$ is the composition of a polynomial $R$ with a nuclear operator $S \circ J$. However, $P$ need not be nuclear. Indeed, the polynomial $P \in \mathcal{P}\left({ }^{2} C[0,1]\right)$ given by

$$
P(f):=\int_{0}^{1} f(t)^{2} d t \quad(f \in C[0,1])
$$

is (Pietsch) integral with values in the scalar field (which has trivially the RadonNikodým property), but $P$ is not nuclear [2, Remark 2.4].

(b) Since $\ell_{1}$ has the Radon-Nikodým property, the operator $A \circ J$ in diagram (5) is also nuclear, and we have

$$
\|A \circ J\|_{\mathrm{PI}}=\|A \circ J\|_{\mathrm{N}} ;
$$

so the inequalities of (6) show that

$$
\|P\|_{\mathrm{PI}}=\inf \|R\|\|A \circ J\|_{\mathrm{N}}\|L\| .
$$

(c) If $F$ has the Radon-Nikodým property, since the operator $S \circ J$ is nuclear in diagram (5), the polynomial $P=S \circ J \circ R$ is compact. This was known, since the Radon-Nikodým property implies the compact range property, and it is proved in $[15$, Theorem 4.10] that $F$ has the compact range property if and only if every Pietsch integral polynomial with values in $F$ is compact. 
As an application, we extend to the polynomial setting the following well-known result [18, Theorem VIII.4.12].

Theorem 3.5. Consider the operators $T \in \mathcal{L}(E, F)$ and $S \in \mathcal{L}(F, Y)$. Then:

(a) if $T$ is integral and $S$ is weakly compact, $S \circ T$ is nuclear;

(b) if $T$ is weakly compact and $S$ is integral, $S \circ T$ is nuclear into $Y^{* *}$.

Using the left integral $\ell_{1}$-factorisation property, we can improve the results of $[\mathbf{1 3}$, Proposition 5].

TheOrem 3.6. Let $P \in \mathcal{P}\left({ }^{k} E, F\right)$ be a polynomial, and let $S \in \mathcal{L}(F, Y)$ and $T \in$ $\mathcal{L}(X, E)$ be operators. Then:

(a) if $P$ is integral and $S$ is weakly compact, $S \circ P$ has the left integral $\ell_{1}$-factorisation property;

(b) if $T$ is integral and $P$ is weakly compact, $P \circ T$ has the left integral $\ell_{1}$-factorisation property;

(c) if $T$ is weakly compact and $P$ is integral, $P \circ T$ is nuclear into $F^{* *}$. below:

Proof. (a) By Theorem 1.2, we can find a factorisation of $j_{F} \circ P$ as in the diagram

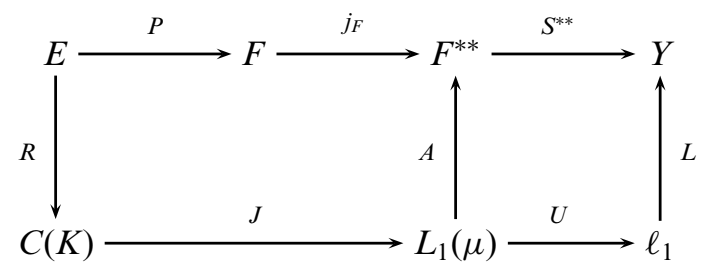

Since $S^{* *} \circ A$ is weakly compact, the comment after Theorem 1.1 yields an $\ell_{1}$ factorisation $S^{* *} \circ A=L \circ U$. Then, letting $Q:=U \circ J \circ R$ which is a Pietsch integral polynomial by Theorem 1.2, we have

$$
S \circ P=S^{* *} \circ j_{F} \circ P=S^{* *} \circ A \circ J \circ R=L \circ U \circ J \circ R=L \circ Q .
$$

(b) We can find an integral factorisation of $T$ as in the diagram below:

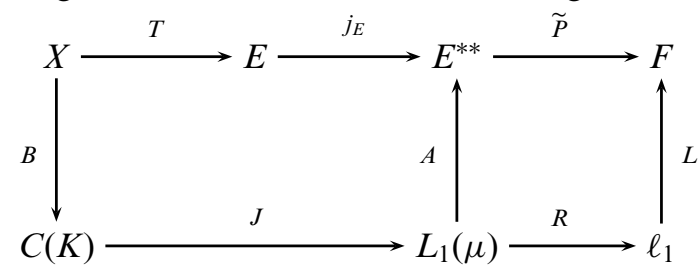

The Aron-Berner extension $\widetilde{P}$ of $P$ is $F$-valued and is itself weakly compact (see [9, Proposition 1.4] and its proof); so $\widetilde{P} \circ A$ is a weakly compact polynomial on $L_{1}(\mu)$. By Remark 2.8, it is left $\ell_{1}$-factorable in the form $\widetilde{P} \circ A=L \circ R$, where $R$ is a polynomial.

Since $J \circ B$ is integral, the polynomial $Q:=R \circ J \circ B$ is (Pietsch) integral [12, Corollary 2.7] and so

$$
P \circ T=\widetilde{P} \circ j_{E} \circ T=\widetilde{P} \circ A \circ J \circ B=L \circ R \circ J \circ B=L \circ Q .
$$

(c) is proved in [13, Proposition 5]. 
ACKNOWLEDGEMENTS. The first author was supported in part by the GNAMPA (Italy). Both authors were supported in part by Dirección General de Investigación, MTM 2006-03531 (Spain). The authors are grateful to the referee for valuable suggestions and for pointing out the references [10] and [22].

\section{REFERENCES}

1. F. Albiac and N. J. Kalton, Topics in Banach space theory, Graduate Texts in Mathematics, vol. 233 (Springer, New York, 2006).

2. R. Alencar, Multilinear mappings of nuclear and integral type, Proc. Am. Math. Soc. 94 (1985), 33-38. $131-138$

3. R. Alencar, On reflexivity and basis for $\mathcal{P}\left({ }^{m} E\right)$, Math. Proc. R. Ir. Acad. 85A (1985),

4. J. M. Ansemil and K. Floret, The symmetric tensor product of a direct sum of locally convex spaces, Studia Math. 129 (1998), 285-295.

5. R. M. Aron, C. Hervés and M. Valdivia, Weakly continuous mappings on Banach spaces, J. Funct. Anal. 52 (1983), 189-204.

6. R. M. Aron and J. B. Prolla, Polynomial approximation of differentiable functions on Banach spaces, J. Reine Angew. Math. 313 (1980), 195-216.

7. F. Blasco, Complementation in spaces of symmetric tensor products and polynomials, Studia Math. 123 (1997), 165-173.

8. G. Botelho and D. M. Pellegrino, Two new properties of ideals of polynomials and applications, Indag. Math. (N.S.) 16 (2005), 157-169. $359-372$

9. D. Carando, Extendible polynomials on Banach spaces, J. Math. Anal. Appl. 233 (1999),

10. D. Carando, V. Dimant and S. Muro, Coherent sequences of Banach ideals of polynomials, to appear in Math. Nachr.

11. D. Carando and S. Lassalle, $E^{\prime}$ and its relation with vector-valued functions on $E, A r k$. Math. 42 (2004), 283-300.

12. R. Cilia, M. D'Anna and J. M. Gutiérrez, Polynomial characterization of $\mathscr{L}_{\infty}$-spaces, J. Math. Anal. Appl. 275 (2002), 900-912.

13. R. Cilia and J. M. Gutiérrez, Nuclear and integral polynomials, J. Austral. Math. Soc. 76 (2004), 269-280.

14. R. Cilia and J. M. Gutiérrez, Extension and lifting of weakly continuous polynomials, Studia Math. 169 (2005), 229-241.

15. R. Cilia and J. M. Gutiérrez, Integral and S-factorizable multilinear mappings, Proc. $R$. Soc. Edinb. 136A (2006), 115-137.

16. R. Cilia and J. M. Gutiérrez, Ideals of integral and $r$-factorable polynomials, to appear in Bol. Soc. Mat. Mexicana.

17. A. Defant and K. Floret, Tensor norms and operator ideals, Mathematics Studies, vol. 176 (North-Holland, Amsterdam, 1993).

18. J. Diestel and J. J. Uhl, Jr., Vector measures, Mathematical Surveys and Monographs, vol. 15 (American Mathematical Society, Providence RI, 1977).

19. S. Dineen, Complex analysis on infinite dimensional spaces, Springer Monographs in Mathematics (Springer, Berlin, 1999).

20. T. Figiel, Factorization of compact operators and applications to the approximation problem, Studia Math. 45 (1973), 191-210.

21. K. Floret, Natural norms on symmetric tensor products of normed spaces, Note Mat. 17 (1997), 153-188.

22. K. Floret, Minimal ideals of $n$-homogeneous polynomials on Banach spaces, Results Math. 39 (2001), 201-217.

23. K. Floret, On ideals of $n$-homogeneous polynomials on Banach spaces, in Topological algebras with applications to differential geometry and mathematical physics (Athens, 1999) (Strantzalos P. and Fragoulopoulou M., Editors) (University of Athens, Athens, 2002), $19-38$.

24. K. Floret and S. Hunfeld, Ultrastability of ideals of homogeneous polynomials and multilinear mappings on Banach spaces, Proc. Am. Math. Soc. 130 (2002), 1425-1435. 
25. M. González and J. M. Gutiérrez, Factorization of weakly continuous holomorphic mappings, Studia Math. 118 (1996), 117-133.

26. W. B. Johnson, Factoring compact operators, Isr. J. Math. 9 (1971), 337-345.

27. J. Lindenstrauss and A. Pełczyński, Absolutely summing operators in $\mathscr{L}_{p}$-spaces and their applications, Studia Math. 29 (1968), 275-326.

28. J. Lindenstrauss and H. P. Rosenthal, The $\mathscr{L}_{p}$-spaces, Isr. J. Math. 7 (1969), 325-349.

29. J. Mujica, Complex analysis in Banach spaces, Mathematics Studies, vol. 120 (NorthHolland, Amsterdam, 1986).

30. W. Rudin, Principles of mathematical analysis (McGraw-Hill, New York, 1976).

31. R. A. Ryan, Applications of topological tensor products to infinite dimensional holomorphy, PhD Thesis (Trinity College, Dublin, 1980).

32. R. A. Ryan, Weakly compact holomorphic mappings on Banach spaces, Pac. J. Math. 131 (1988), 179-190.

33. R. A. Ryan, Introduction to tensor products of Banach spaces, Springer Monographs in Mathematics (Springer, London, 2002).

34. I. Villanueva, Integral mappings between Banach spaces, J. Math. Anal. Appl. 279 (2003), 56-70.

35. P. Wojtaszczyk, Banach spaces for analysts, Cambridge Studies in Advanced Mathematics, vol. 25 (Cambridge University Press, Cambridge, UK 1991). 